\title{
Strategic Trading
}

\section{AND \\ LEARNING ABOUT LIQUIDITY*}

\author{
Harrison Hong $^{\dagger}$ \\ Stanford University \\ Sven Rady $\ddagger$ \\ Stanford University, University of Munich and CEPR
}

First Draft: November 1998

This Draft: February 1999

\begin{abstract}
We develop a multi-period model of strategic trading in an asset market where traders are uncertain about market liquidity. In our model, informed traders strategically trade against competitive market makers to exploit their short-lived private information. Unlike market makers, informed traders do not know whether the liquidity ("noise") trades are generated from a distribution with high or low variance. Instead, informed traders have to learn about liquidity from past prices. We find the following. (1) Prices that deviate markedly from the forecast of terminal asset value based on public news tend to lead to revisions of informed traders' beliefs in favor of the low liquidity state. (2) This revision in beliefs results in less aggressive trading on private information by informed traders. (3) In turn, informational efficiency and trading volume are dependent on the path of prices. (4) Moreover, learning about liquidity also has interesting effects on the unconditional properties of optimal strategic trading policies.
\end{abstract}

\footnotetext{
${ }^{*}$ We would like to thank Anat Admati, Lanier Benkard, Larry Blume, Darrell Duffie, Paul Pfleiderer, Ken Singleton, Jeremy Stein, Dimitri Vayanos, Jiang Wang, Ingrid Werner, Jeff Zwiebel and seminar participants at the London School of Economics, Oxford University and the Stanford Finance Lunch for helpful comments and discussions.

${ }^{\dagger}$ Graduate School of Business, Stanford University, Stanford, CA 94305-5015; tel.: (+1) 650725 6939; e-mail: Hong_Harrison@gsb.stanford.edu.

${ }^{\ddagger}$ Graduate School of Business, Stanford University, Stanford, CA 94305-5015, and Department of Economics, University of Munich, D-80539 Munich, Germany; tel.: (+49) 892180 5642; e-mail: Sven.Rady@lrz.uni-muenchen.de.
} 


\section{Introduction}

Large traders such as institutional investors (e.g. mutual funds) play an important role in many financial markets. To begin with, institutional ownership of common stocks is quite substantial. For instance, Gompers and Metrick (1999) report that at the end of 1996, large institutions held discretionary control over more than half of the U.S. equity market. Furthermore, institutions account for a substantial fraction of trading volume in a number of exchanges. Schwartz and Shapiro (1992) estimate that in 1990 institutions accounted for over seventy percent of the trading volume among the New York Stock Exchange, the London Stock Exchange and the Tokyo Stock Exchange. Importantly, their trades can significantly affect price dynamics. Holthausen, Leftwich and Mayers (1990) find a price impact of about one percent for the largest buy and sell trades for randomly selected NYSE firms in 1983, while Keim and Madhavan (1996) find a price impact of around eight percent for block trades on small NYSE, AMEX and NASDAQ firms from 1985-1992. ${ }^{1}$ Hence, a better understanding of the economics of asset trading by large traders can help deepen our understanding of many financial markets.

An influential framework for thinking about asset trading by large traders is developed in Kyle (1985). There, a risk-neutral, informed trader (or an "insider") trades with risk-neutral market makers to speculate on private information about asset payoffs. The informed trader submits market orders taking into account the effect of trades on prices. Market makers trade with the informed trader because of the presence of liquidity ("noise") traders, and set prices efficiently conditional on the order flow. Kyle shows that the informed trader trades so as to reveal information slowly until the time when the private information is made public. This basic result has been extended in a number of dimensions. ${ }^{2}$

\footnotetext{
${ }^{1}$ See also Kraus and Stoll (1972), Scholes (1972), Holthausen, Leftwich and Mayers (1987), Hausman, Lo and MacKinlay (1992), and Chan and Lakonishok (1993).

${ }^{2}$ Admati (1991) and O'Hara (1995) provide surveys of this growing literature on strategic trading. Relatedly, a number of other papers in the market-microstructure literature have shown that strategic trading is an important part of explanations for a number of empirical findings regarding intra-day return and volume patterns (see, e.g., Admati and Pfleiderer (1988), Foster and Vishwanathan (1990)). We discuss these extensions in more detail in Section 7 below.
} 
This view of asset trading by large traders, however, is not wholly consistent with a number of findings in the empirical literature on institutional trading (see, e.g., Chan and Lakonishok (1993, 1995), Keim and Madhavan (1995)). While there is evidence that institutional traders execute their trades slowly (presumably to reduce price impact), there are a number of more basic stylized facts about institutional trading that do not fit easily into the rational trading models following the Kyle framework. First, if large traders had such a significant informational advantage over market makers (as is presupposed in the basic variants of the Kyle model), then they ought to outperform the market or various passive benchmarks. This, however, does not appear to be the case. ${ }^{3}$ Second and relatedly, this "implementation shortfall" may be due to the costliness of executing trades (see, e.g., Perold (1988)). Indeed, many institutions expend considerable resources on trading facilities and personnel engaged in acquiring information not about asset payoffs per se but also about the trading environment and the various costs of executing large trades. There is not an explicit role for such behavior in Kyle like models since large traders for the most part have perfect information about the trading environment. For instance, an important determinant of the cost of trading - the supply of liquidity (or variance of noise trades) — is known to all.

But in reality, large traders often face incomplete information regarding the supply of liquidity. First, traders generally do not have information on market makers' inventory fluctuations or other characteristics of order flow which would affect market liquidity. Also, market liquidity may vary substantially across trading days for a variety of reasons (e.g. episodic shutdowns of online brokerage systems like Schwab or E-Trade). Such shocks are probably more directly observable to market markers who are constantly observing order flow than large traders who participate in the market on a less frequent basis.

Motivated by these observations, we develop a multi-period, equilibrium model of strategic trading in which there are shocks to the supply of liquidity that are observed

\footnotetext{
${ }^{3}$ Numerous studies have documented portfolio managers' inability to outperform various passive benchmarks, despite considerable effort to analyze and select stocks (see, e.g., Jensen (1969), Fama (1991) and Carhart (1997)).
} 
by market makers but unobservable to informed traders. As such, the informed traders face uncertainty regarding the price impact of their trades since they have incomplete information about the true state of liquidity. However, they can extract valuable information about the true state of liquidity by learning from past market prices. This learning about liquidity on the part of informed traders comes closer to behavior observed in the real world on the part of institutional traders. In contrast, informed traders in the basic Kyle framework have no need to learn from past prices since they have superior information to market makers. More importantly, since informed traders' beliefs about the true state of liquidity change over time depending on the path of prices, past prices then help to predict how aggressively informed traders trade and, in turn, trading volume and the informational efficiency of prices.

More specifically, our model is similar to a version of the Kyle model, in which risk-neutral informed traders strategically trade against risk-neutral and competitive market makers to exploit their short-lived private information (see, e.g., Kyle (1985) and Admati and Pfleiderer (1988)). Noise trades are generated from either a distribution with high variance or a distribution with low variance. The true distribution from which noise trades are drawn is known to market makers but imperfectly observed by informed traders. ${ }^{4}$ In general, informed traders can learn about the true state of liquidity from past prices since the price distributions under high versus low variance of liquidity trades have different expected returns and volatility conditional on the informed traders' information set.

Below, we fully characterize the dynamics of the informed traders' beliefs about the state of liquidity. One result that emerges is the dependence of the revision of these beliefs on past prices. Intuitively, averaging over realizations of private signals, the sensitivity of price to order flow will depend on the true liquidity state -it will be higher when the variance of noise trades is small (i.e., when the market is less liquid). The price in any trading round is simply the forecast of terminal asset value based on previously released public news plus a component related to the order flow in that

\footnotetext{
${ }^{4}$ This assumption is a simple way of capturing large traders' incomplete information about aspects of the order flow better known to market makers. See Section 2.4 below for a more thorough discussion.
} 
round. All else equal, informed traders (on average) tend to revise their beliefs in favor of the low liquidity state when past prices deviate significantly from the forecasted terminal asset value based on public news.

In turn, the dependence of revisions in beliefs on past prices implies that strategic trading, informational efficiency and trading volume will also be path dependent. When informed traders are uncertain about the liquidity in the market, they trade as if the price impact were an average of those in the high and low variance states, weighted by their beliefs about the likelihood of each state occurring. The more confident they are that the true state is low, the lower the liquidity they expect and the less aggressively they trade on their private information. If recent price deviations from fundamentals have been large, then informed traders tend to revise their beliefs in favor of a low liquidity market and end up trading less aggressively on their private information. In turn, extreme deviations of price from fundamentals in one period tend to be followed by lower informational efficiency and lower trading volume in the subsequent period.

Beyond the dependence of strategic trading on the path of past prices, we also find that liquidity uncertainty has interesting effects on the time path of strategic trading. ${ }^{5}$ We identify several effects. One effect is for informed traders to trade gingerly early on and to trade more aggressively over time as their uncertainty about the variance of liquidity trades is resolved gradually. Another effect is that the low variance state may be so bad (i.e. so illiquid) in comparison to the high variance state that on average informed traders end up trading less aggressively over time. We characterize how these effects interact to determine the time path of strategic trading. Of course, liquidity uncertainty is most likely to have persistent effects on the time path when learning is slow. We show that this is the case when the difference between the high and the low variance of noise trades is sufficiently small and the number of informed traders sufficiently large.

Our model is related to a large literature on strategic trading following Kyle (1985). We carefully compare our model to those in this literature and other related models

\footnotetext{
${ }^{5}$ By the time path of strategic trading, we mean the following: how does the aggressiveness of trades on private information vary unconditionally over time?
} 
in Section 7. The big difference between our model and most in this literature is that we consider the consequences of liquidity uncertainty for dynamic patterns in strategic trading. We emphasize the effects of learning from past prices (in particular, about non-fundamental information), which are absent from these models. As such, most of our results are very different from those derived in the literature. ${ }^{6}$

In the following section, we develop a simple dynamic model to capture these ideas. Equilibrium trading and pricing strategies are derived in Section 3. The emphasis of Section 4 is on understanding how traders learn from prices about market liquidity. In Section 5, we discuss the time variation in strategic trading induced by learning about liquidity. In Section 6, we draw out the empirical implications of our model for strategic trading. We contrast our work with related models in Section 7 . Section 8 concludes. All proofs are in the Appendix.

\section{The Model}

In this section, we analyse a simple model of strategic trading. We consider a setup similar to that of Kyle (1985). One risky security is traded by three types of traders: $N$ informed, risk-neutral traders who possess identical private information about the liquidation value of the risky security, liquidity ("noise") traders who trade for idiosyncratic or liquidity reasons, and competitive, risk-neutral market makers.

\subsection{Timing and Information Structure}

The single asset is traded over a span of time equal to $T$ trading rounds. It is assumed that the ex post liquidation value of the asset at the end of round $T$ is exogenously given by

$$
v=\sum_{t=1}^{T} v_{t}
$$

\footnotetext{
${ }^{6}$ One paper from this literature which shares our goals is Lindsey (1992). He develops a dynamic model with long-lived private information in which the low variance state can only be the extreme case of zero variance (i.e., no noise trades whatsoever). We will carefully contrast our model to Lindsey's in Section 7. It turns out that in his model there is little learning in the sense that we describe. As such, the results that we derive are very different from his.
} 
where $v_{t}$ for $t=1, \ldots, T$ are independently distributed normal random variables each having a mean zero and variance $\sigma_{v}^{2}$. Any trader holding a share of the asset at the end of round $T$ receives a liquidating dividend of $v$ dollars. At the beginning of round $t, v_{t}$ is observed by the informed traders. At the end of round $t, v_{t}$ becomes public information to all market participants. In other words, the informed traders have a short-lived informational advantage in each round. One can think of $v_{t}$ as public news, which the informed traders get to peek at the period before it is released. By the end of round $T$, the value of the asset will be known by all.

The quantity traded by noise traders in round $t$, denoted by $u_{t}$, is drawn from a normal distribution with mean zero and a variance $\sigma_{u}^{2}$ that nature determines prior to any trading. This variance can assume two values, $\sigma_{0}^{2}$ or $\sigma_{1}^{2}$, with $\sigma_{1}^{2}>\sigma_{0}^{2}>0$. The variables $v_{1}, \ldots, v_{T}$ are independent of the noise trades and, conditional on $\sigma_{u}^{2}$, the variables $u_{1}, \ldots, u_{T}$ are independent as well.

In each round, trading takes place in two steps as follows. In step one, the exogenous values of $v_{t}$ and $u_{t}$ are realized and the $N$ informed traders choose the quantities they want to trade, $x_{n, t}(n=1, \ldots, N)$. Before doing so, they observe $v_{t}$. But the informed traders do not know $u_{t}$ when placing an order, nor do they know from which of the two possible distributions it was drawn. In step two, the market makers determine the price $p_{t}$ at which they trade the quantity necessary to clear the market. When doing so, they observe the total order flow $y_{t}=\sum_{n=1}^{N} x_{n, t}+u_{t}$ but not the $x_{n, t}$ or $u_{t}$ separately. However, unlike the informed traders, they do know from which distribution $u_{t}$ was drawn. That is, they know the realization of $\sigma_{u}^{2}$.

\subsection{Pricing}

Competitive, risk neutral market makers determine the price in period $t$ based on the history of public information, $v_{1}, \ldots, v_{t-1}$, and on past and current order flows, $y_{1}, \ldots, y_{t}$. Let $V_{t}=\left(v_{1}, v_{2}, \ldots, v_{t}\right)$ and $Y_{t}=\left(y_{1}, y_{2}, \ldots, y_{t}\right)$. The zero expected profit condition implies that $p_{t}$, the price set in period $t$ by the market makers, satisfies

$$
p_{t}=\mathrm{E}\left[v \mid \sigma_{u}^{2}, V_{t-1}, Y_{t}\right]
$$


For future convenience, let $P_{t}=\left(p_{1}, \ldots, p_{t}\right)$ be the history of prices.

\subsection{Informed Trading and Learning}

Now consider an informed trader's decision. We assume that each informed trader, in each period $t$, chooses a trade $x_{n, t}$ to maximize the expected time $t$ profits, which are given by

$$
\mathrm{E}\left[x_{n, t}\left(v-p_{t}\right) \mid V_{t}, P_{t-1}\right] .
$$

Because of the nature of the short-lived private information, issues related to the timing of informed trading, which are important in Kyle (1985), do not arise here. ${ }^{7}$ In our model, periods are only linked through the informed traders' updating of beliefs about the current variance of noise trades.

In the equilibrium that we consider below, the revelation of $v_{t}$ makes the trades $x_{n, t}$, and hence total informed order flow, publicly known at the end of round $t$. Each informed trader then uses this information to update his belief about the variance of noise trades according to Bayes' Law. Suppose that informed traders begin trading round $t$ with the common belief assigning probability $\pi_{t}$ to the event that $\sigma_{u}^{2}=\sigma_{1}^{2}$. At the end of round $t$, they update this belief on the basis of the price $p_{t}$ given the orders $x_{n, t}$ that they placed. This updated belief $\pi_{t+1}$ is then taken into trading round $t+1$, and the process repeats itself. At the end of each round, the market makers know everything that the informed traders know, so they can infer the informed traders' updated belief.

\subsection{Comments on the Model}

An important assumption of our model is that market makers have more information regarding the variance of liquidity trades than informed traders. This assumption is a simple way to model the natural asymmetry in information between market makers and traders regarding certain characteristics of the order flow. For instance, liquidity trades may include interdealer trading motivated by risk sharing (see, e.g., Reiss

\footnotetext{
${ }^{7}$ Moreover, by assumption, we are shutting down active learning (optimal experimentation) by informed traders. In other words, they optimize profits without taking into account that their actions could affect the informativeness of prices. We will revisit this point in Section 2.4.
} 
and Werner (1998)). One can also think of the private information about variances as information about discretionary liquidity trading (see, e.g., Admati and Pfleiderer (1988)). It is reasonable to assume that market makers have better information about the distribution of such trades. ${ }^{8}$

A potentially more problematic assumption is the one to shut down optimal experimentation by the informed traders. Given the "myopic" objective function (3), the informed traders do not take into account the fact that their trades will generate information for them. That is, we preclude the possibility of the informed traders engaging in active learning or optimal experimentation. ${ }^{9}$ While it would be interesting to consider this effect, we can justify our assumption on at least two grounds. Even if we gave our traders a forward-looking objective function, the resulting equilibrium would be qualitatively similar to the one we have constructed. This is because every trade has the potential to reveal some information, so the traders have no incentive to engage in large deviations from their myopic best response. ${ }^{10}$ Additionally, the deviations of traders' policies under optimal experimentation from their myopic ones are less prominent if there are more traders as there is an incentive to free-ride on the information generated by others. ${ }^{11}$ As such, our results are likely to remain qualitatively similar.

Other possible extensions of our model would be to consider long-lived private information and diverse information. While there is little doubt that considering these richer information structures would enhance our model and potentially allow us to address more issues, our basic results are likely not to be affected. In fact, the learning problem of informed traders is unaffected by whether their private information is longlived, while the possibility of diverse information complicates the learning problem

\footnotetext{
${ }^{8}$ Like many other rational expectations models, we assume that competitive market makers do not sell or compete on their private information. One can think of many different rationales behind this; see the many related models of trading on private information following Grossman (1976).

${ }^{9}$ See Leach and Madhavan (1993) for a study of optimal experimentation in a financial markets context. These authors consider optimal experimentation by a monopolistic market maker.

${ }^{10}$ In the terminology of the economics literature on optimal experimentation, this means that there are no "confounding" (i.e. completely uninformative) actions in our model; cf. Easley and Kiefer (1988). For examples of single-agent decision problems where there are such actions and where the resulting optimal strategy is qualitatively very different from the myopic one, see Kiefer (1989) and Keller and Rady (1999).

${ }^{11}$ See Bolton and Harris (1999) for an analysis of this effect in a model of strategic experimentation.
} 
without altering its main properties. Neither of these richer information structures would therefore change the fact that informed traders can learn from past prices about non-fundamental information (i.e. the variance of liquidity trades). So future strategic trading and informational efficiency would again be path dependent on past prices, as in the simple model we present here.

\section{Equilibrium}

\subsection{Prices and Trading Strategies}

We characterize the unique linear equilibrium of the trading game. In a linear equilibrium, the informed traders place orders $x_{n, t}=\beta_{n, t} v_{t}$ and the market makers employ a pricing rule of the form

$$
p_{t}= \begin{cases}\sum_{j=1}^{t-1} v_{j}+\lambda_{0, t} y_{t} & \text { if } \sigma_{u}^{2}=\sigma_{0}^{2} \\ \sum_{j=1}^{t-1} v_{j}+\lambda_{1, t} y_{t} & \text { if } \sigma_{u}^{2}=\sigma_{1}^{2}\end{cases}
$$

Our notation conforms with that in Kyle (1985). Here, $\lambda_{k, t}(k=0,1)$ measures the responsiveness of price to order flow when the noise trades are drawn from the distribution with variance $\sigma_{k}^{2}$. As news $v_{t}$ about the terminal value of the asset gets revealed over time, the expected liquidation value of the asset changes. Market makers adjust the price in response to current order flow, which conveys some of the informed traders' short-lived private information.

Given the pricing rule (4), the informed traders' uncertainty about the variance of noise trades translates directly into price impact uncertainty. The average $\lambda_{t}=$ $\pi_{t} \lambda_{1, t}+\left(1-\pi_{t}\right) \lambda_{0, t}$ measures the informed traders' expected price impact. As in Kyle (1985), the inverse $1 / \lambda_{t}$ captures the market depth dimension of market liquidity: this

is the order size that, from the perspective of an informed trader, will on average move the price by one unit. Of course, actual market depth depends on the true variance of noise trade and is measured by $1 / \lambda_{k, t}$ with $k=0$ or 1 .

The following result describes equilibrium strategies as a function of current beliefs. To state it, we define

$$
\bar{\sigma}_{t}^{2}=\pi_{t} \sigma_{1}^{2}+\left(1-\pi_{t}\right) \sigma_{0}^{2}
$$


This is the variance of uninformed order flow that informed traders expect in trading round $t$ given that their belief at the beginning of the trading round is $\pi_{t}$.

Lemma 3.1 The dynamic trading game has a unique linear equilibrium. In this equilibrium, all informed traders submit identical market orders, $x_{t}=\beta_{t} v_{t}$, expecting to achieve trading profits of $\beta_{t} v_{t}^{2} /(N+1)$ per trader. Market makers use a pricing strategy as in (4) with the informed traders' expected price impact given by

$$
\lambda_{t}=\pi_{t} \lambda_{1, t}+\left(1-\pi_{t}\right) \lambda_{0, t}=\frac{1}{(N+1) \beta_{t}} .
$$

The equilibrium $\beta_{t}, \lambda_{0, t}$ and $\lambda_{1, t}$ are

$$
\beta_{t}=\beta\left(\pi_{t}\right)=\frac{\sqrt{\gamma_{t}}}{\sqrt{N} \sigma_{v}}
$$

and

$$
\lambda_{k, t}=\lambda_{k}\left(\pi_{t}\right)=\frac{\sqrt{N \gamma_{t}} \sigma_{v}}{N \gamma_{t}+\sigma_{k}^{2}}
$$

where $\gamma_{t}=\gamma\left(\pi_{t}\right)$ is the unique positive root of the quadratic equation

$$
N \gamma^{2}+\left[\sigma_{0}^{2}+\sigma_{1}^{2}-(N+1) \bar{\sigma}_{t}^{2}\right] \gamma-\sigma_{0}^{2} \sigma_{1}^{2}=0
$$

The equilibrium law of motion for beliefs is given by

$$
\begin{aligned}
& \pi_{t+1}=\left[1+\frac{1-\pi_{t}}{\pi_{t}} \frac{\left(N \gamma_{t}+\sigma_{0}^{2}\right) \sigma_{1}}{\left(N \gamma_{t}+\sigma_{1}^{2}\right) \sigma_{0}} \times\right. \\
& \exp \left(\frac { \sigma _ { 1 } ^ { 2 } - \sigma _ { 0 } ^ { 2 } } { 2 N \gamma _ { t } \sigma _ { 0 } ^ { 2 } \sigma _ { 1 } ^ { 2 } \sigma _ { v } ^ { 2 } } \left[\left(\sigma_{0}^{2} \sigma_{1}^{2}-N^{2} \gamma_{t}^{2}\right)\left(p_{t}-\sum_{j=1}^{t-1} v_{j}\right)^{2}\right.\right. \\
&\left.\left.\left.+2 N^{2} \gamma_{t}^{2} v_{t}\left(p_{t}-\sum_{j=1}^{t-1} v_{j}\right)-N^{2} \gamma_{t}^{2} v_{t}^{2}\right]\right)\right]^{-1}
\end{aligned}
$$

for $t=1, \ldots, T-1$.

\subsection{Simple Properties of the Equilibrium}

First, we point out some properties of the equilibrium price function. Since the market makers always know the true distribution from which the liquidity trades are drawn, 
the price set by the market makers is more sensitive to order flow (and the market is less deep) when the variance of noise trades is low: $\lambda_{0, t}>\lambda_{1, t}$. Furthermore, competition between the risk-neutral market makers ensures that, conditional on the true variance of noise trades, price is an unbiased estimator. So by taking the expectation over that variance, we see that price is also an unbiased forecast unconditionally. These results are stated formally in the following proposition.

Proposition 3.1 In the equilibrium with price impact uncertainty, the price is more sensitive to order flow when the variance of noise trades is low. However, price is always an unbiased forecast of the liquidation value:

$$
p_{t}=E\left[v \mid V_{t-1}, p_{t}\right]
$$

We next point out a few basic properties of the informed traders' equilibrium strategies. To develop some intuition, consider first the special case where the informed traders have full information on the state of the variance of noise trades, i.e. $\pi_{t}=k \in\{0,1\}$. Then $\gamma_{t}=\sigma_{k}^{2}$ and $\beta_{t}=\sigma_{k} /\left(\sqrt{N} \sigma_{v}\right)$, which is exactly the equilibrium strategy for the $N$-player one-period Kyle model with commonly known variance $\sigma_{k}^{2}$. For non-degenerate beliefs, equation (7) shows that the informed traders behave as if they were in an $N$-player one-period Kyle model with known variance $\gamma_{t}$. In this sense, $\gamma_{t}$ is the "certainty equivalent variance" for the informed traders. A second useful interpretation of the variable $\gamma_{t}$ emerges when we calculate the variance of the informed order flow: $\operatorname{Var}\left[N \beta_{t} v_{t} \mid V_{t-1}, P_{t-1}\right]=N^{2} \beta_{t}^{2} \sigma_{v}^{2}=N \gamma_{t}$. Thus, the variance of informed order flow is proportional to $\gamma_{t}$, the factor of proportionality being the number of informed traders. Finally, we note from equation (6) that the trading aggressiveness $\beta_{t}$ of an informed trader is inversely related to the expected price impact of his trades, $\lambda_{t}$. This generalizes a well-known property of the standard Kyle model to the case of price impact uncertainty.

We have the following intuitive results on informed investors' trading strategies. First, $\gamma_{t}$ and $\beta_{t}$ increase in $\pi_{t}$. That is,

Proposition 3.2 The informed traders trade more aggressively as they become more confident that the variance of noise trades is high. 
When informed traders trade more aggressively, the informed order flow becomes an increasingly important component of total order flow. The trading strategies of informed traders, given by $\beta_{t}$, change over time as they learn from past prices and update their beliefs $\pi_{t}$ about the distribution that generates the noise trades. The market makers condition their pricing rule on the variance of noise trades as well as the beliefs of the informed traders. Consequently, the equilibrium price functions, characterized by their slopes $\lambda_{k, t}$, also change over time. How beliefs are updated, i.e. equation (10), will be the focus of the next section.

\section{Learning about Liquidity}

In this section, we study how informed investors learn about the variance of liquidity trades over time. While informed traders do have private information on stock payoffs, they cannot perfectly infer the true variance of liquidity trades from prices since they do not observe the noise trades. However, the price distributions under high versus low variance of liquidity trades will in general have different expected returns and volatility conditional on the informed traders' private information; so informed traders can extract valuable information about the likelihood of each state from market prices.

\subsection{The Basics of Updating}

More precisely, given the belief $\pi_{t}$ held at the beginning of trading round $t$, and conditional on the realization of $v_{t}$ and the true variance $\sigma_{u}^{2}=\sigma_{k}^{2}$, the price innovation

$$
z_{t}=p_{t}-\sum_{j=1}^{t-1} v_{j}=\lambda_{k, t}\left(N \beta_{t} v_{t}+u_{t}\right)
$$

is normally distributed with mean

$$
\mathrm{E}\left[z_{t} \mid \pi_{t}, v_{t}, \sigma_{k}^{2}\right]=\lambda_{k, t} N \beta_{t} v_{t}=\frac{N \gamma_{t}}{N \gamma_{t}+\sigma_{k}^{2}} v_{t}=: \mu_{k}\left(\pi_{t}\right) v_{t}
$$

and variance

$$
\operatorname{Var}\left[z_{t} \mid \pi_{t}, v_{t}, \sigma_{k}^{2}\right]=\lambda_{k, t}^{2} \sigma_{k}^{2}=\frac{N \gamma_{t} \sigma_{v}^{2} \sigma_{k}^{2}}{\left(N \gamma_{t}+\sigma_{k}^{2}\right)^{2}}=: \Sigma_{k}\left(\pi_{t}\right)^{2} .
$$

We call $z_{t}$ a price innovation in the sense that given the forecasted fundamentals $\sum_{j=1}^{t-1} v_{j}$ or the fair price for the asset entering period $t, z_{t}$ can be thought of as the deviation 
or innovation from fundamentals. We denote the corresponding density functions by $f_{k}\left(z_{t} \mid \pi_{t}, v_{t}\right)(k=0,1)$. It follows that the likelihood ratio is given by

$$
\begin{aligned}
\ell\left(z_{t} \mid \pi_{t}, v_{t}\right) & =\frac{f_{1}\left(z_{t} \mid \pi_{t}, v_{t}\right)}{f_{0}\left(z_{t} \mid \pi_{t}, v_{t}\right)} \\
& =\frac{\Sigma_{0}\left(\pi_{t}\right)}{\Sigma_{1}\left(\pi_{t}\right)} \exp \left(-\frac{1}{2}\left[\frac{z_{t}-\mu_{1}\left(\pi_{t}\right) v_{t}}{\Sigma_{1}\left(\pi_{t}\right)}\right]^{2}+\frac{1}{2}\left[\frac{z_{t}-\mu_{0}\left(\pi_{t}\right) v_{t}}{\Sigma_{0}\left(\pi_{t}\right)}\right]^{2}\right) .
\end{aligned}
$$

By Bayes' rule, the belief held after observing $v_{t}$ and $z_{t}$ is

$$
\pi_{t+1}=\frac{\pi_{t} f_{1}\left(z_{t} \mid \pi_{t}, v_{t}\right)}{\pi_{t} f_{1}\left(z_{t} \mid \pi_{t}, v_{t}\right)+\left(1-\pi_{t}\right) f_{0}\left(z_{t} \mid \pi_{t}, v_{t}\right)}=\left[1+\frac{1-\pi_{t}}{\pi_{t}} \frac{1}{\ell\left(z_{t} \mid \pi_{t}, v_{t}\right)}\right]^{-1},
$$

which increases with the likelihood ratio. In particular, we have $\pi_{t+1}>\pi_{t}$ if and only if $\ell\left(z_{t} \mid \pi_{t}, v_{t}\right)>1$, i.e. if and only if the observed price is more likely to have been generated from the distribution associated with the high variance of noise trades. With some straightforward algebra, the expression in equation (16) reduces to the right hand side of the updating equation (10) in Lemma 3.1.

\subsection{Dynamics of Beliefs}

The dynamics of beliefs depend on a number of factors: among them are the observed price innovation $z_{t}$, the private information $v_{t}$, the difference between the two possible variances $\sigma_{1}^{2}$ and $\sigma_{0}^{2}$, and the number $N$ of traders. We will address the impact of each of these factors in this sub-section, which aims to provide some qualitative insights into the dynamics behind equation (10).

Given the belief $\pi_{t}$ and the private information $v_{t}$, the price signal $z_{t}$ is drawn from one of two possible normal distributions. As $\mu_{1}\left(\pi_{t}\right)<\mu_{0}\left(\pi_{t}\right)$, these two distributions have different means (unless $v_{t}=0$, which is a null event). The informed traders thus expect to see price innovations closer to zero when the market is deeper. If the two possible price distributions had the same variance, informed traders would therefore put more weight on the high variance state whenever they saw a price innovation close to zero. Examination of (14) shows, however, that the two distributions will in general have different variances.

Small absolute price innovations will still be evidence in favor of the deeper market if a deeper market has a smaller price variance. Yet, as we shall see shortly, this need not 
be the case. Nor is a deeper market necessarily associated with a higher price variance. While this would hold true if the market makers used the same pricing strategy in both states, they actually choose a flatter pricing strategy when the variance of noise trades is high $\left(\lambda_{0, t}>\lambda_{1, t}\right)$. As the variance of price innovations is the product of the squared slope of the pricing strategy and the variance of noise trades, it is not clear, without further analysis, in what state the variance of price innovations will be higher. As a consequence, a small absolute price innovation, for example, is not necessarily evidence for a deeper market.

To determine which of the two possible price distributions has the higher variance we have to compare the difference in the squared slopes of pricing strategies, $\lambda_{0, t}^{2}-\lambda_{1, t}^{2}$, with the given difference in the variances of noise trades, $\sigma_{1}^{2}-\sigma_{0}^{2}$. It is straightforward to show that $\lambda_{0, t}^{2}-\lambda_{1, t}^{2}$ decreases monotonically in $N \gamma_{t}$, the variance of informed order flow. This is very intuitive: a growing variance of informed order flow means that orders from the informed traders become a larger component of total order flow, while the orders from noise traders become less important; consequently, the market makers' incentive to vary their pricing strategy according to the true variance of noise trades diminishes. The higher the variance of informed order flow, therefore, the less pronounced is the flattening of the market makers' pricing strategy when the true variance of noise trades is high, and the higher is the price variance in the high liquidity state relative to the price variance in the low liquidity state. More precisely, the ranking of these two price variances depends on whether $N \gamma_{t}$ exceeds $\sigma_{0} \sigma_{1}$ or not. We thus need to distinguish between two cases.

The first case is when $N \gamma_{t}<\sigma_{0} \sigma_{1}$. In this instance, $\Sigma_{1}\left(\pi_{t}\right)<\Sigma_{0}\left(\pi_{t}\right)$, and so the deeper market is associated with a smaller variance of price innovations. The quadratic in the exponent of the likelihood ratio (15) is then strictly concave in $z_{t}$, with a global maximum at

$$
\frac{\Sigma_{0}\left(\pi_{t}\right)^{2} \mu_{1}\left(\pi_{t}\right)-\Sigma_{1}\left(\pi_{t}\right)^{2} \mu_{0}\left(\pi_{t}\right)}{\Sigma_{0}\left(\pi_{t}\right)^{2}-\Sigma_{1}\left(\pi_{t}\right)^{2}} v_{t}=\frac{N^{2} \gamma_{t}^{2}}{N^{2} \gamma_{t}^{2}-\sigma_{0}^{2} \sigma_{1}^{2}} v_{t}=: \mu\left(\pi_{t}\right) v_{t}
$$

Thus, the informed traders update the stronger in favor of the state where the market is deeper, the closer the realized innovation is to $\mu\left(\pi_{t}\right) v_{t}$. Price innovations far off this 
mark are more likely to have been generated from the price distribution with higher variance, $\Sigma_{0}\left(\pi_{t}\right)^{2}$, hence lead the traders to update in favor of $\sigma_{0}^{2}$.

The second case is when $N \gamma_{t}>\sigma_{0} \sigma_{1}$. In this instance, $\Sigma_{1}\left(\pi_{t}\right)>\Sigma_{0}\left(\pi_{t}\right)$, and so the deeper market is associated with a higher variance of price innovations. The quadratic is now strictly convex in $z_{t}$, with a global minimum at $\mu\left(\pi_{t}\right) v_{t}$. Price innovations far off this mark are more likely to stem from the price distribution with variance $\Sigma_{1}\left(\pi_{t}\right)^{2}$, hence lead the traders to update in favor of $\sigma_{1}^{2}$.

The borderline between these two cases is given by the equality $N \gamma_{t}=\sigma_{0} \sigma_{1}$. By equation (9), this is equivalent to $\sigma_{0}^{2}+\sigma_{1}^{2}-(N+1) \bar{\sigma}_{t}^{2}-(N-1) \sigma_{0} \sigma_{1}=0$, which holds if and only if the current belief is

$$
\pi_{t}=\frac{\sigma_{1}-N \sigma_{0}}{(N+1)\left(\sigma_{1}-\sigma_{0}\right)}
$$

In this instance, $\Sigma_{1}\left(\pi_{t}\right)^{2}=\Sigma_{0}\left(\pi_{t}\right)^{2}=\sigma_{0} \sigma_{1} /\left(\sigma_{0}+\sigma_{1}\right)^{2}$, and the updating equation simplifies to

$$
\pi_{t+1}=\left[1+\frac{1-\pi_{t}}{\pi_{t}} \exp \left(\frac{\sigma_{1}^{2}-\sigma_{0}^{2}}{\sigma_{0} \sigma_{1} \sigma_{v}^{2}} v_{t}\left[z_{t}-\frac{1}{2} v_{t}\right]\right)\right]^{-1} .
$$

This is strictly decreasing in $z_{t}$ if $v_{t}>0$, and strictly increasing if $v_{t}<0$, in accordance with our earlier statement that for identical price variances, a price innovation close to zero is indicative of a deeper market. Of course, if $v_{t}=0$, then the two possible distributions for $z_{t}$ are identical, and there is no updating at all.

Note that, whatever the position of $N \gamma_{t}$ relative to $\sigma_{0} \sigma_{1}$, a larger (in absolute value) realization of the private information $v_{t}$ allows the informed traders to learn more about the true variance of noise trades. This is because an increase in $\left|v_{t}\right|$ drives the means of the two possible price distributions further apart while keeping their variances unchanged, and so makes the observed prices more informative.

Note also that the right-hand side of (18) equals $\frac{1}{2}$ for $N=1$, decreases in $N$ and $\sigma_{0}$, and increases in $\sigma_{1}$. If $\sigma_{1} \leq N \sigma_{0}$ (which requires at least two traders, and will always hold for sufficiently large $N$ ) the right-hand side of (18) is non-positive, so $N \gamma_{t}>\sigma_{0} \sigma_{1}$ at all beliefs $\pi_{t}$. As the number of traders or the spread between the two possible variances of uninformed order flow grows, therefore, we see that for a larger range of beliefs, the state with a high variance of noise trades (and thus a deeper market) is 
characterized by more variable equilibrium prices. In this range, a very high or very low price innovation $z_{t}$ is evidence of a deeper market, hence reason for the informed traders to revise $\pi_{t}$ upward.

\subsection{Revisions of Beliefs and Past Prices}

In this sub-section, we consider a simpler case in which we integrate $\pi_{t+1}$ with respect to $v_{t}$. In other words, we consider updating by an observer who starts with the belief $\pi_{t}$ and observes $z_{t}$, but not $v_{t}$. This is an important and empirically relevant case because the perspective of the outside observer will be the appropriate one when we consider how past price realizations affect future trading behavior and informational efficiency.

Conditional on the true variance being $\sigma_{u}^{2}=\sigma_{k}^{2}$, such an observer anticipates the equilibrium price innovation, $z_{t}=\lambda_{k, t}\left(N \beta_{t} v_{t}+u_{t}\right)$, to be normally distributed with

mean zero and variance $\hat{\Sigma}_{k}\left(\pi_{t}\right)^{2}=N \gamma_{t} \sigma_{v}^{2} /\left(N \gamma_{t}+\sigma_{k}^{2}\right)$. Note that this variance is unambiguously smaller in the state where the market is deeper: $\hat{\Sigma}_{1}\left(\pi_{t}\right)<\hat{\Sigma}_{0}\left(\pi_{t}\right)$. This is because now the variance of price innovations is the product of the squared slope of the market makers' pricing strategy and the variance of total order flow (not just uninformed order flow as in the previous sub-section). Thus, the variance of price innovations has two components: one stemming from informed order flow $\left(\lambda_{k, t}^{2} N \gamma_{t}\right)$, the other from uninformed order flow $\left(\lambda_{k, t}^{2} \sigma_{k}^{2}\right)$. When the variance of informed order flow is small $\left(N \gamma_{t}<\sigma_{0} \sigma_{1}\right)$, then both these components are smaller in the state where the market is deeper: $\lambda_{1, t}^{2} N \gamma_{t}<\lambda_{0, t}^{2} N \gamma_{t}$ and $\lambda_{1, t}^{2} \sigma_{1}^{2}<\lambda_{0, t}^{2} \sigma_{0}^{2}$. When the variance of informed order flow is large $\left(N \gamma_{t} \geq \sigma_{0} \sigma_{1}\right)$, the "informed component" is still smaller in the state where the market is deeper, but the "uninformed component" is larger in this state: $\lambda_{1, t}^{2} N \gamma_{t}<\lambda_{0, t}^{2} N \gamma_{t}$ and $\lambda_{1, t}^{2} \sigma_{1}^{2} \geq \lambda_{0, t}^{2} \sigma_{0}^{2}$. Yet precisely because the variance of informed order flow is large, the "informed component" dominates, and we obtain the same ranking of the two possible price variances as before. When we do not condition on the private information $v_{t}$, therefore, a deeper market unambiguously means a lower variance of equilibrium prices.

Consequently, an observer who sees a price innovation close to zero will put more weight on the state where the variance of uninformed order flow is high. Conversely, a 
very high or very low price innovation is ascribed to a lack of market depth, and more weight is put on the state where the variance of uninformed order flow is low. In fact, the same arguments as above imply that in response to seeing an innovation $z_{t}$, the observer updates his belief to

$$
\hat{\pi}_{t}=\left[1+\frac{1-\pi_{t}}{\pi_{t}} \sqrt{\frac{N \gamma_{t}+\sigma_{0}^{2}}{N \gamma_{t}+\sigma_{1}^{2}}} \exp \left(\frac{\left(\sigma_{1}^{2}-\sigma_{0}^{2}\right) z_{t}^{2}}{2 N \gamma_{t} \sigma_{v}^{2}}\right)\right]^{-1}
$$

which is strictly decreasing in $\left|z_{t}\right|$.

Note that $\hat{\pi}_{t}$ is the conditional mean of the informed traders' updated belief: $\hat{\pi}_{t}=$ $\mathrm{E}\left[\pi_{t+1} \mid \pi_{t}, z_{t}\right]$. So our last result suggests that after a small price innovation, an informed trader will tend to be more confident that liquidity is high. To formalize this idea, we use the conditional median of the updated belief as a measure of its central tendency. ${ }^{12}$

Proposition 4.1 After a small absolute price innovation, an informed trader is more likely to revise his beliefs in favor of the high liquidity state than in favor of the low liquidity state.

More precisely, the median of $\pi_{t+1}$ conditional on $\pi_{t}$ and $z_{t}$ exceeds $\pi_{t}$ if and only if $\left|z_{t}\right|$ is below some threshold. The conditional probability of the event $\pi_{t+1}>\pi_{t}$ then exceeds one half.

The implications of this result for strategic trading, informational efficiency and trading volume will be spelled out in Sections 5.1-5.3.

\subsection{Speed of Learning and Convergence in the Long Run}

In this sub-section, we first consider the speed of learning, and then the asymptotics of learning when the number of trading rounds becomes large. The results we obtain will be useful in characterizing the empirical content of our model as far as the time path of strategic trading is concerned.

\footnotetext{
${ }^{12}$ The main advantage of using the median rather than the mean will become apparent when we ask how trading aggressiveness varies with past price observations. As trading aggressiveness $\beta_{t+1}$ depends monotonically but nonlinearly on the belief $\pi_{t+1}$, it is difficult to translate statements about the mean of $\pi_{t+1}$ into statements about the mean of $\beta_{t+1}$. Doing this for the medians is trivial.
} 
The speed of learning, i.e., how much adjustment in beliefs one should expect to see in any given trading round, depends on how much information about the variance of noise trades the informed traders can extract from equilibrium prices. This in turn depends on key factors such as the difference between the high and the low variance of noise trades, and the number of informed traders.

A measure of the difference between the two possible price distributions, and hence of the information content of observed prices, is the entropy of one price distribution relative to the other. To keep matters simple, we proceed as in Section 4.3 and average over the realizations of the private information $v_{t}$. Suppose that the true variance of noise trades is $\sigma_{1}^{2}$. The entropy of interest is then defined as

$$
e\left(\pi_{t}\right)=\int_{-\infty}^{\infty} f_{1}\left(z_{t} \mid \pi_{t}\right) \ln \frac{f_{1}\left(z_{t} \mid \pi_{t}\right)}{f_{0}\left(z_{t} \mid \pi_{t}\right)} d z_{t}
$$

where $f_{k}\left(z_{t} \mid \pi_{t}\right)$ is the density function for the price innovation $z_{t}$ if $\sigma_{u}^{2}=\sigma_{k}^{2}$. The entropy $e\left(\pi_{t}\right)$ is always non-negative, and equals zero if and only if the two density functions coincide. Moreover, a higher entropy means a higher information content of observed prices, and hence a faster revision of beliefs. ${ }^{13}$

As in Section 4.3, $f_{k}\left(z_{t} \mid \pi_{t}\right)$ is a normal density with mean zero and variance $\hat{\Sigma}_{k}\left(\pi_{t}\right)^{2}=N \gamma_{t} \sigma_{v}^{2} /\left(N \gamma_{t}+\sigma_{k}^{2}\right)$. It is thus straightforward to calculate

$$
e\left(\pi_{t}\right)=\frac{1}{2}\left[\frac{\hat{\Sigma}_{1}\left(\pi_{t}\right)^{2}}{\hat{\Sigma}_{0}\left(\pi_{t}\right)^{2}}-\ln \frac{\hat{\Sigma}_{1}\left(\pi_{t}\right)^{2}}{\hat{\Sigma}_{0}\left(\pi_{t}\right)^{2}}-1\right]
$$

and perform comparative statics for the speed of learning.

We first consider the effect of widening the difference between the high and the low variance of liquidity trades.

Proposition 4.2 The speed of learning increases with the difference between the two possible variances of noise trades.

This finding is intuitive given the results in Section 4.3. The larger the spread between the two possible variances of noise trades, the larger is the spread between the

\footnotetext{
${ }^{13}$ In a simple example with binary uncertainty about the fundamental asset value, binary signals (sale or purchase) and i.i.d. trades, O'Hara (1995, pp. 82-86) shows that beliefs converge exponentially at a rate equal to the entropy. Our setup here is more complicated insofar as the entropy itself changes as beliefs change, but the basic relationship between speed of learning and entropy carries over.
} 
corresponding price volatilities, and the easier it becomes to distinguish the two states (given that both price distributions have mean zero). As a consequence, beliefs tend to move more in response to observations.

Another interesting parameter that affects the speed of learning is the number of informed traders in the market, $N$. We find the following.

Proposition 4.3 The speed of learning decreases with the number of informed traders.

Again, this is fairly intuitive. As the number of informed traders grows, the distribution of noise trades becomes less important to the overall order flow and the formation of prices. As a consequence, the difference between the two possible price distributions shrinks, and price observations reveal less about the variance of noise trades.

Next, we analyze the behavior of beliefs as the number of trading rounds grows large, i.e., $T \rightarrow \infty$. Since the noise trades each period are drawn from the same distribution with a variance $\sigma_{u}^{2}$ determined before trading begins, and each trading round reveals some information unless $v_{t}=0$ (which is a zero-probability event), it is natural to expect that, given a sufficiently large number of trading rounds, the informed traders will get arbitrarily close to certainty about $\sigma_{u}^{2}$. This intuition need not hold in general: the equilibrium could be badly behaved in a way that allows for incomplete learning even in the very long run. We can confirm, however, that our equilibrium is such that the above intuition holds.

Proposition 4.4 As the number of trading rounds becomes large, the informed traders learn the true variance of noise trades with probability one.

The implications of the above results for the time path of strategic trading are addressed in Section 5.4.

\section{Time Variation in Strategic Trading}

Having established an understanding of the dynamics of beliefs, we turn to the implications of learning by informed traders for their trading strategies and in turn for informational efficiency and trading volume. 


\subsection{Past Prices and Strategic Trading}

The first implication of our model is that past prices help predict how aggressively informed traders trade on their private information. To see this, recall from Proposition 3.2 that as the informed investors' confidence in the high variance of liquidity trades increases, they trade more aggressively. From the analysis in Section 4.3 and more precisely Proposition 4.1, we know that after small absolute price innovations, beliefs tend to be revised in favor of the high liquidity state. Putting these two results together, we immediately obtain the following proposition.

Proposition 5.1 After a small absolute price innovation in a given period, informed traders tend to trade more aggressively on their private information in the following period.

The intuition for this result is clear. Small absolute price innovations tend to indicate a deeper market, and informed traders take advantage of a deeper market by trading more aggressively.

\subsection{Past Prices and Informational Efficiency}

When the informed traders face uncertainty about the variance of liquidity trades, they are no longer able to trade exactly the "right" amount, and informational efficiency depends on the extent to which informed traders over- or underestimate market depth. If they overestimate it, they will trade too aggressively and reveal too much information. If they underestimate market depth, they will trade too gingerly and reveal too little information. After seeing the price, the outside observer can draw inferences as to which of the two scenarios is more likely, and how much information has been incorporated in the price.

In this regard, a measure of informational efficiency in trading round $t$ is

$$
q_{t}=\operatorname{Var}\left[v_{t} \mid \pi_{t}, V_{t-1}, p_{t}\right]=\operatorname{Var}\left[v_{t} \mid \pi_{t}, z_{t}\right] .
$$

Higher informational efficiency means a lower "residual variance" $q_{t}$. This is the appropriate measure for an observer whose information set is the same as that of an 
informed trader, except that he has no privileged information about $v_{t}$ at the beginning of each trading period. From the revelation of $v_{t}$ at the end of each trading period (and the prior belief of the informed traders at $t=1$ ), this observer can reconstruct the sequence of beliefs $\pi_{t}(t=2, \ldots, T)$ held by the informed traders at the beginning of each trading round.

It is well known that in the Kyle model, the informed traders trade in such a way as to reveal exactly the fraction $N /(N+1)$ of their private information, i.e., the above measure of informational efficiency equals $\sigma_{v}^{2} /(N+1)$. Interestingly, under certainty, informational efficiency is constant, and it does not depend at all on the (known) variance of liquidity trades. The main intuition behind this result is that the informed traders trade just aggressively enough to take advantage of any additional variance.

With uncertainty about liquidity, however, informed traders are unable to fine-tune their trades in this way, and informational efficiency becomes a stochastic variable depending on beliefs and price innovations. In fact, when the absolute price innovation $\left|z_{t}\right|$ is large, it is quite likely, given the analysis in Section 4.3, that the market is less liquid. So the informed traders probably overestimated the variance of noise trades (and in turn market depth) and traded too aggressively. Hence, when $\left|z_{t}\right|$ is large, it is likely that the informed traders revealed too much information and so prices are more informationally efficient than in the certainty benchmark. Conversely, when $\left|z_{t}\right|$ is small, it is quite likely that the informed traders underestimated the variance of noise trades (and in turn market liquidity) and traded too gingerly. Hence, when $\left|z_{t}\right|$ is small, the informed traders probably did not reveal enough information and so prices are less informationally efficient than if there were complete information about liquidity trades. The following proposition confirms this.

Proposition 5.2 With price impact uncertainty, informational efficiency of prices, as measured in (22), depends on the price and is strictly increasing in the absolute magnitude of the price innovation.

More precisely, the proof shows that there is a cutoff level $\left|\bar{z}_{t}\right|$ such that for absolute price innovations above $\left|\bar{z}_{t}\right|$, informational efficiency is higher than under complete 
information, i.e., $q_{t}<\sigma_{v}^{2} /(N+1)$; whereas for absolute innovations below $\left|\bar{z}_{t}\right|$, informational efficiency is lower than in the benchmark, i.e., $q_{t}>\sigma_{v}^{2} /(N+1)$. The proof of the proposition also shows that average informational efficiency is exactly as under complete information: $\mathrm{E}\left[q_{t} \mid \pi_{t}\right]=\sigma_{v}^{2} /(N+1)$.

\subsection{Past Prices and Trading Volume}

We next consider the effects of liquidity uncertainty and learning on trading volume. Following Admati and Pfleiderer (1988), we use the standard deviation of order flow to measure the contribution of each group of traders to the total trading volume. As before, we adopt the perspective of an observer whose information set is the same as that of an informed trader, except that he has no privileged information about the underlying asset value at the beginning of the trading period. This leads us to define

$$
\Sigma_{t}^{I}=\sqrt{\operatorname{Var}\left[\sum_{n=1}^{N} x_{n, t} \mid \pi_{t}, V_{t-1}\right]},
$$

$\Sigma_{t}^{U}=\sqrt{\operatorname{Var}\left[u_{t} \mid \pi_{t}, V_{t-1}\right]}, \Sigma_{t}^{M}=\sqrt{\operatorname{Var}\left[y_{t} \mid \pi_{t}, V_{t-1}\right]}$, and $\Sigma_{t}=\Sigma_{t}^{I}+\Sigma_{t}^{U}+\Sigma_{t}^{M}$. In other words, $\Sigma_{t}^{I}$ and $\Sigma_{t}^{U}$ measure the expected volume of trading of the informed and liquidity traders, respectively, and $\Sigma_{t}^{M}$ measures the expected trading done by the market maker. The total expected trading volume, $\Sigma_{t}$, is simply the sum of the individual components.

Unlike the case of complete information in which past prices are uninformative as to the level of volume, past prices do help predict future levels of trading volume when there is liquidity uncertainty. To see this, recall from Section 5.1 that past prices help predict the intensity of trading on private information — small absolute price innovations tend to be followed by increased confidence in the high liquidity state, hence more aggressive trading in the subsequent round. It follows easily then that the expected trading volume generated by informed traders, $\Sigma_{t}^{I}$, increases also following small price innovations. By definition, increased confidence in the high liquidity state also implies higher expected trading volume from uninformed traders, $\Sigma_{t}^{U}$. Finally, the market maker has to accomodate more trades as the expected order flows from informed and uninformed traders increase. So, $\Sigma_{t}^{M}$ will tend to increase after small price realizations as well. Hence, we have the following proposition. 
Proposition 5.3 Small absolute price realizations tend to be followed by higher expected trading volume.

\subsection{The Time Path of Strategic Trading}

Up to this point, we have primarily emphasized the path dependence of strategic trading, informational efficiency and trading volume on past prices. In this sub-section, we consider the implications of the model for the time path of trading. To this end, we want to isolate the pure effect of uncertainty about liquidity. We fix an expected level of liquidity $\bar{\sigma}_{t}^{2}$ and vary the spread between the two possible variances of noise trades. The higher this spread, the more uncertain informed traders are about liquidity. We have the following result.

Proposition 5.4 As the informed traders' uncertainty about liquidity increases (holding expected liquidity constant), they expect a higher price impact of their trades, trade less aggressively, and expect lower profits.

In fact, the proof shows that, given an expected variance of noise trades $\bar{\sigma}_{t}^{2}$, the certainty equivalent variance $\gamma_{t}$ is strictly decreasing in the difference $\sigma_{1}^{2}-\sigma_{0}^{2}$. In view of equation (7), therefore, the aggressiveness of informed trading, measured by $\beta_{t}$, also decreases in $\sigma_{1}^{2}-\sigma_{0}^{2}$. In particular, informed traders trade less aggressively than if the variance of noise trades were $\bar{\sigma}_{t}^{2}$ for sure.

This uncertainty effect, which is already discussed in Lindsey (1992), is easy to understand. Suppose that the informed traders are uncertain about liquidity, but use the trading strategy $x_{t}=\bar{\beta}_{t} v_{t}$ with $\bar{\beta}_{t}=\sqrt{\bar{\sigma}_{t}^{2}} /\left(\sqrt{N} \sigma_{v}\right)$ that would be the equilibrium strategy if the variance of noise trades were commonly known to be $\bar{\sigma}_{t}^{2}$. The market makers, who know the true variance $\sigma_{u}^{2}$, determine the slope of their pricing strategy by estimating the asset value on the basis of the order flow. By the projection formula for normal variables, this slope coefficient turns out to be strictly convex in $\sigma_{u}^{2}$. By Jensen's inequality, therefore, the expected slope $\lambda_{t}=\pi_{t} \lambda_{1, t}+\left(1-\pi_{t}\right) \lambda_{0, t}$ exceeds $\bar{\lambda}_{t}=\sqrt{N} \sigma_{v} /\left[(N+1) \sqrt{\bar{\sigma}_{t}^{2}}\right]$, which would be the equilibrium slope if the variance of noise trades were $\bar{\sigma}_{t}^{2}$ for sure. Since it is the expected slope that determines the price 
response anticipated by the informed traders, they face higher expected costs to trading, and are thus better off trading less aggressively. At the same time, higher expected trading costs mean lower expected profits.

We first note that the uncertainty effect identified in Proposition 5.4 becomes less important over time: trading aggressiveness resembles more and more that in the hypothetical "certainty benchmark" equilibrium where the variance of liquidity trades in round $t$ is commonly known to be $\bar{\sigma}_{t}^{2}$. This is formalized in the next result, which is an immediate corollary of Proposition 4.4.

Proposition 5.5 The difference $\bar{\beta}_{T}-\beta_{T}$, which measures the effect of uncertainty about liquidity on trading behavior, converges to zero almost surely as the number of trading rounds becomes large.

That is, as the informed traders' uncertainty about market liquidity diminishes over time, these traders tend to trade more aggressively relative to the benchmark $\bar{\beta}_{t}=$ $\sqrt{\bar{\sigma}_{t}^{2}} /\left(\sqrt{N} \sigma_{v}\right)$. At the same time, market depth tends to increase relative to the benchmark, and so do the traders' expected profits.

While the informed traders tend to trade more aggressively over time relative to the certainty benchmark, this does in general not imply that they can expect to trade more aggressively in absolute terms. In fact, the upward trend from the diminishing uncertainty effect could be more than offset by a downward level effect should the true state be the one where noise trades are less volatile. Thus, the process $\beta_{t}$ is generally not a submartingale relative to the informed traders' information set, i.e., it is in general not true that $\mathrm{E}\left[\beta_{t+1} \mid \pi_{t}, V_{t-1}, P_{t-1}\right] \geq \beta_{t}$.

For a small number of informed traders and a small spread between $\sigma_{1}^{2}$ and $\sigma_{0}^{2}$, however, we are able to show the submartingale property for $\beta_{t}$.

Proposition 5.6 Let $N=1$ or 2 , and suppose that $\sigma_{1}^{2} \leq 3 \sigma_{0}^{2} / N$. Then the informed traders expect to trade more aggressively and earn higher per-period profits as time passes.

The stated condition on $\sigma_{0}^{2}, \sigma_{1}^{2}$ and $N$ guarantees convexity of $\beta_{t}$ in $\pi_{t}$. As the belief process is a martingale from the informed traders' point of view $\left(\mathrm{E}\left[\pi_{t+1} \mid \pi_{t}, V_{t-1}, P_{t-1}\right]=\right.$ 
$\pi_{t}$ ), the result then follows from Jensen's inequality. In other words, the condition ensures that the equilibrium shares a crucial property with single-agent optimization problems: a positive value of information. This positive value manifests itself in the convexity of the expected profit function (which is proportional to $\beta_{t}$ here). While the value of information in single-agent learning problems is always positive, it can be negative when there is competition and information spillover. In fact, if $N>2$, then the expected profit (and $\beta_{t}$ ) as a function of $\pi_{t}$ always has a concave segment at the right end of the unit interval - the value of information is negative when informed traders are very optimistic about market depth.

The second role of the above condition is to ensure that the spread between the two possible variances is small, implying small level effects. Beliefs can converge to zero but this will not have an impact large enough on average to overcome the upward drift from the resolution of uncertainty. Conversely, when $\sigma_{1}^{2}>3 \sigma_{0}^{2} / N$, the low liquidity state can be so illiquid that informed traders with sufficiently optimistic beliefs must expect to be trading at their most aggressive early on.

Note that markets with slow learning are more likely to experience persistent effects from liquidity uncertainty. We saw in Section 4.4 that learning will be the slower, the smaller the spread between the two possible variances and the greater the number of informed traders. When the spread between the variances becomes very small and the number of informed traders very large, however, the importance of these effects in terms of prices and profits diminishes. Hence, our results on the time path of strategic trading are most likely to be relevant in markets with a variance spread and a number of informed traders that both lie in an intermediate range.

\section{Empirical Implications}

In this section, we draw out the empirical implications of our model. Without belaboring the point, our model matches a number of basic stylized facts about strategic trading that are absent from many of the existing models. First, from the results in Section 5.4, liquidity uncertainty decreases the profitability of informed trading. That 
informed traders do not have such a significant advantage over all others is more in accord with the empirical evidence that presumably informed mutual fund managers find it hard to beat various passive benchmarks. Of course, there can be many other reasons for the poor performance - for instance, there could be multiple informed traders who compete their informational advantage away. However, it seems to us that a lack of information about the costs of trading is an ex ante reasonable factor since there is evidence that large traders expend considerable resources to gather information on their trading environment. In this vein, the idea that informed traders can learn about nonfundamental information from past prices fits nicely with evidence that institutions do look to prices for information about the cost of trading.

Beyond this general observation, our model generates a number of testable implications. The most basic is how past prices affect future strategic trading. Extreme price innovations, reflecting a market with low liquidity, will lead to less aggressive trading by informed traders in the next period. One could test this prediction using data sets on institutional trades similar to those in Keim and Madhavan (1995) and Chan and Lakonishok $(1993,1995)$.

For instance, Keim and Madhavan use data on buyer and seller initiated trades for the period of January 1991 to March 1993. This data includes the date when the trading decision was made, the desired number of shares in the order at the time of the trading decision with a buy-sell indicator, the number of broker releases per order, the duration before orders were filled and the choice of order type (active market orders or more passive limit orders). Keim and Madhavan study various aspects of trading behavior motivated by models such as Kyle (1985) and more generic trading behavior such as feedback trading.

Here, we propose that one could use this data to get a reasonable measure of aggressiveness of trading on private information (i.e., estimate $\beta_{t}$ using desired order size or order type) and test the prediction of the model by regressing this measure on a measure of illiquidity derived from past prices (higher moments or some measure of deviation from forecasted fundamentals). Of course, one would need to control for a variety of factors, but seeing how future strategic trading depends on past prices would 
be interesting.

In a similar vein, it would not be hard to implement the predictions regarding the path dependence of trading volume on past prices. The result on informational efficiency is of course more difficult to test, though not entirely impossible with more data. The results on the time path of trading are also potentially testable but much harder; one might need to appeal to methods developed in Ellison and Mullin (1997), who identify events in which there is likely to be speculation.

\section{Comparison with Related Models}

The goal of our paper is broadly related to the large literature on strategic trading following Kyle (1985) - to understand the nature of strategic trading and its effects on price and volume. To mention just a few papers from this literature: Back (1992) extends Kyle's results for more general distributions of asset payoffs; Holden and Subrahmanyam (1992) consider the case of many informed traders who have the same information; Foster and Vishwanathan (1996) and Back, Cao and Willard (1998) assume many informed traders who have different pieces of information.

For the most part, this literature has maintained the assumption that the parameters of the model (such as the variance of noise trades) are known to all. Thus, there is no scope for traders to learn about non-fundamental information from prices, and past prices have little effect on future decisions of the agents in these models. The existence of such a link between past prices and future actions is perhaps the most distinguishing feature of our model. This link generates many empirical implications that are not obtainable under the assumption of perfect information about liquidity.

As alluded to in the introduction, Lindsey (1992) comes closest to our model. He also considers a market where informed traders do not know the variance of noise trades whereas market makers do. Unlike us, he develops a dynamic model with long-lived private information in which the low variance state can only be the extreme case of zero variance (i.e., no noise trades whatsoever). ${ }^{14}$ In each period, there is some probability

\footnotetext{
${ }^{14} \mathrm{He}$ also develops a static model in which the low variance state need not be zero. This static version pre-dates the static version of our model.
} 
that the low variance state is drawn - everyone finds out immediately and the informed traders make no trading profits after that point. So, the informed trader always knows that he is in the high variance state as long as the trading game continues. There is little learning from past prices. The model is like Kyle (1985) except that there is a certain probability that the game will end each period and informed traders lose out on further speculative opportunities. Hence, informed traders tend to trade more aggressively on their private information than in the Kyle benchmark.

In our model, the low variance state is not necessarily zero variance, so there is scope for genuine learning from past prices. Hence, our results on learning about the variance of noise trades are absent from Lindsey (1992), as are all of our implications regarding the path dependence of strategic trading, informational efficiency and trading volume on past prices.

More broadly, our model is similar to a few other models that consider parameter uncertainty of a similar form. Forster and George (1992) consider a static model in which market makers have better information regarding liquidity trades. They examine the consequences of anonymity of liquidity trading on various welfare measures. Kumar and Seppi (1994) examine a model of arbitrage in index futures, in which the precision of heterogeneous signals received by market makers at different geographic locations is private information. And more recently, Gervais (1997) and Spiegel and Subrahmanyam (1999) consider models in which market markers not only lack information on an informed trader's signal about the mean of the asset value but also have to infer the ex-ante value (variance) of his private information. While the set-ups of these models share some similarities to ours, their focus and results are entirely different from ours.

\section{Conclusion}

In this paper, we develop a model to study the effects of price impact uncertainty on the optimal trading strategies of large traders as well as the equilibrium feedback to prices. In our model, risk-neutral informed traders strategically trade against riskneutral competitive market makers to exploit short-lived private information. Unlike 
market makers, these informed traders have incomplete information about the distribution from which liquidity ("noise") trades are drawn. As a result, they face uncertainty about the price impact of their trades (i.e. market liquidity). They optimally take into account this uncertainty in their trades and learn about market liquidity from past prices.

To summarize, we find the following. First, prices that deviate markedly from the forecast of terminal asset value based on public news tend to lead to revisions of informed traders' beliefs in favor of the low liquidity state. Furthermore, this revision in beliefs results in less aggressive trading on private information by informed traders. In turn, informational efficiency and trading volume are dependent on the path of prices. Finally, learning about liquidity has interesting effects on the unconditional properties of optimal strategic trading policies.

Even though the model is highly stylized, it does exhibit an attractive property: past prices affect future market liquidity and trading strategies. This feature is missing in most of the existing models of strategic trading. Hence, a careful study of the dynamics may yield more insights into return and trading patterns. Moreover, we can also incorporate richer dynamics by allowing for the variance of liquidity trades to change over time. For instance, we can allow the variance of liquidity trades to follow a two-state Markov chain and study the dynamics generated in this changing environment.

Our focus is purely on the speculative motive for trade on the part of large traders. In reality, large traders probably trade to both speculate and hedge. This dual trading motive is captured in a number of papers such as Admati and Pfleiderer (1988), Foster and Vishwanathan (1990), Seppi (1990), and Vayanos (1998). Additionally, we have ignored the dynamics in the unwinding of large positions, which is an important problem encountered by large institutions. This problem is better addressed by Bertsimas and Lo (1998) who study the dynamic strategies of large traders who have a fixed time horizon to complete a trade. To fully understand the importance of uncertainty about market liquidity, a model should incorporate these other motives for trade as well. 


\section{Appendix}

\section{A Proofs}

\section{Proof of Lemma 3.1}

Given the informed traders' strategies $x_{n, t}=\beta_{n, t} v_{t}(n=1, \ldots, N)$ in trading round $t$, the market makers can calculate the conditional expectation of $v_{t}$ as

$$
\mathrm{E}\left[v_{t} \mid \sigma_{u}^{2}, \sum_{n=1}^{N} \beta_{n, t} v_{t}+u_{t}=y_{t}\right]=\frac{\operatorname{Cov}\left[v_{t}, \sum_{n=1}^{N} \beta_{n, t} v_{t}+u_{t} \mid \sigma_{u}^{2}\right]}{\operatorname{Var}\left[\sum_{n=1}^{N} \beta_{n, t} v_{t}+u_{t} \mid \sigma_{u}^{2}\right]} y_{t} .
$$

Thus, if $\sigma_{u}^{2}=\sigma_{k}^{2}$ with $k \in\{0,1\}$, the market makers use the pricing rule $p_{t}=\sum_{j=1}^{t-1} v_{j}+$ $\lambda_{k, t} y_{t}$ where

$$
\lambda_{k, t}=\frac{\sum_{n=1}^{N} \beta_{n, t} \sigma_{v}^{2}}{\left(\sum_{n=1}^{N} \beta_{n, t}\right)^{2} \sigma_{v}^{2}+\sigma_{k}^{2}} .
$$

Taking this pricing rule and the linear strategies of other informed traders as given, informed trader $n$ 's objective in round $t$ is to maximize

$$
\begin{aligned}
\mathrm{E} & {\left[x_{n, t}\left(v-p_{t}\right) \mid V_{t}, P_{t-1}\right] } \\
& =\mathrm{E}\left[x_{n, t}\left(v_{t}+\sum_{j=t+1}^{T} v_{j}-\lambda_{k, t}\left[x_{n, t}+\sum_{m \neq n} \beta_{m, t} v_{t}+u_{t}\right]\right) \mid V_{t}, P_{t-1}\right] \\
& =x_{n, t}\left(v_{t}-\lambda_{t}\left[x_{n, t}+\sum_{m \neq n} \beta_{m, t} v_{t}\right]\right)
\end{aligned}
$$

with

$$
\lambda_{t}=\mathrm{E}\left[\lambda_{k, t} \mid V_{t}, P_{t-1}\right]=\mathrm{E}\left[\lambda_{k, t} \mid V_{t-1}, P_{t-1}\right]=\pi_{t} \lambda_{1, t}+\left(1-\pi_{t}\right) \lambda_{0, t} .
$$

(Since $\mathrm{E}\left[\lambda_{k, t} u_{t} \mid V_{t}, \sigma_{u}^{2}=\sigma_{k}^{2}\right]=0$ for $k=0,1$, we have $\mathrm{E}\left[\lambda_{k, t} u_{t} \mid V_{t}\right]=0$.) This expression for expected profits has the following interpretation. Informed traders face incomplete information about the type of market maker they are trading with, where "the type" is given by $\lambda_{k, t}$. In a linear equilibrium, they effectively trade with the average type, which is $\lambda_{t}$ as given in (A.4).

The first-order condition yields $x_{n, t}=\left[1-\lambda_{t} \sum_{m \neq n} \beta_{m, t}\right] v /\left[2 \lambda_{t}\right]$, hence $\beta_{n, t}=$ $\left[1-\lambda_{t} \sum_{m \neq n} \beta_{m, t}\right] /[2 \lambda(\pi)]$ or $1=\lambda_{t}\left[2 \beta_{n, t}+\sum_{m \neq n} \beta_{m, t}\right]=\lambda_{t}\left[\beta_{n, t}+\sum_{m=1}^{N} \beta_{m, t}\right]$. This implies that $\beta_{n, t}$ is the same for all informed traders and equal to

$$
\beta_{t}=\frac{1}{(N+1) \lambda_{t}}
$$

By (A.3), therefore, each informed trader's expected profit in trading round $t$ (conditional on $\left.v_{t}\right)$ equals $\left[\beta_{t} v_{t}\right]\left(v_{t}-\lambda_{t}\left[N \beta_{t} v_{t}\right]\right)=\beta_{t} v_{t}^{2} /(N+1)$. 
Using (A.4) and (A.2), we can rewrite (A.5) as

$$
\beta_{t}=\frac{1}{N+1}\left(\pi_{t} \frac{N \beta_{t} \sigma_{v}^{2}}{N^{2} \beta_{t}^{2} \sigma_{v}^{2}+\sigma_{1}^{2}}+\left(1-\pi_{t}\right) \frac{N \beta_{t} \sigma_{v}^{2}}{N^{2} \beta_{t}^{2} \sigma_{v}^{2}+\sigma_{0}^{2}}\right)^{-1} .
$$

Simplifying this equation, we see that it is a quartic in $\beta_{t}$, with only the even powers of $\beta_{t}$ appearing. Writing $\gamma=N \beta_{t}^{2} \sigma_{v}^{2}$, we find that (A.6) transforms into the quadratic equation (9). As the left-hand side of (9) is negative at $\gamma=0$, this equation has a unique positive root. Since a negative $\beta_{t}$ would imply a negative $\lambda_{t}$ in contradiction to the informed traders' second-order condition, we then obtain (7) and (8). This is the unique linear equilibrium.

The updating equation (10) is derived in Section 4.

\section{Proof of Proposition 3.1}

The claim that $\lambda_{0, t}>\lambda_{1, t}$ follows directly from equation (8).

By the definition of equilibrium, $p_{t}=\mathrm{E}\left[v \mid \sigma_{u}^{2}, V_{t-1}, y_{t}\right]$. As $p_{t}=\sum_{j=1}^{t-1} v_{j}+\lambda_{k, t} y_{t}$ when $\sigma_{u}^{2}=\sigma_{k}^{2}(k=0$ or 1$)$, conditioning on $\left(\sigma_{u}^{2}, V_{t-1}, y_{t}\right)$ is equivalent to conditioning on $\left(\sigma_{u}^{2}, V_{t-1}, p_{t}\right)$, so $p_{t}=\mathrm{E}\left[v \mid \sigma_{u}^{2}, V_{t-1}, p_{t}\right]$. This in turn implies $p_{t}=\mathrm{E}\left[v \mid V_{t-1}, p_{t}\right]$.

\section{Proof of Proposition 3.2}

Equation (A.6) characterises $\beta_{t}$ as the unique fixed point of the function

$$
F_{t}(\beta)=\frac{1}{N+1}\left(\pi_{t} \frac{N \beta \sigma_{v}^{2}}{N^{2} \beta^{2} \sigma_{v}^{2}+\sigma_{1}^{2}}+\left(1-\pi_{t}\right) \frac{N \beta \sigma_{v}^{2}}{N^{2} \beta^{2} \sigma_{v}^{2}+\sigma_{0}^{2}}\right)^{-1} .
$$

As $F_{t}(\beta)$ increases in $\pi_{t}$, so does the fixed point $\beta_{t}$, and with it $\gamma_{t}$.

\section{Proof of Proposition 4.1}

We want to show that the median of $\pi_{t+1}$ conditional on $\pi_{t}$ and $z_{t}$ exceeds $\pi_{t}$ when $\left|z_{t}\right|$ is sufficiently small. By continuity, it suffices to show this for $z_{t}=0$. In this case, the likelihood ratio simplifies to

$$
\ell\left(0 \mid \pi_{t}, v_{t}\right)=\frac{\left(N \gamma_{t}+\sigma_{1}^{2}\right) \sigma_{0}}{\left(N \gamma_{t}+\sigma_{0}^{2}\right) \sigma_{1}} \exp \left(\frac{N \gamma_{t}}{2}\left[\frac{1}{\sigma_{0}^{2}}-\frac{1}{\sigma_{1}^{2}}\right] \frac{v_{t}^{2}}{\sigma_{v}^{2}}\right) .
$$

The random variable $v_{t}^{2} / \sigma_{v}^{2}$ has a $\chi^{2}(1)$ distribution, with median $m$ lying between 0.45493 and 0.45494 . The median of $\ell\left(0 \mid \pi_{t}, v_{t}\right)$ is

$$
\frac{\left(N \gamma_{t}+\sigma_{1}^{2}\right) \sigma_{0}}{\left(N \gamma_{t}+\sigma_{0}^{2}\right) \sigma_{1}} \exp \left(\frac{N \gamma_{t}}{2}\left[\frac{1}{\sigma_{0}^{2}}-\frac{1}{\sigma_{1}^{2}}\right] m\right)
$$

and we are done if we can show that it always exceeds 1. 
To this end, we consider the expression

$$
L=\frac{\left(A+\sigma_{1}^{2}\right) \sigma_{0}}{\left(A+\sigma_{0}^{2}\right) \sigma_{1}} \exp \left(\frac{A}{2}\left[\frac{1}{\sigma_{0}^{2}}-\frac{1}{\sigma_{1}^{2}}\right] m\right)
$$

for arbitrary $A>0$. Clearly, $L \rightarrow 1$ as $\sigma_{1} \rightarrow \sigma_{0}$. It is therefore enough to prove that $L$ is strictly increasing in $\sigma_{1}$. Now, straightforward computation shows that the partial derivative of $L$ with respect to $\sigma_{1}$ has the same sign as the quadratic $Q=$ $A^{2} m+A(1-m) \sigma_{1}^{2}+\sigma_{1}^{4}$, which is obviously positive.

\section{Proof of Proposition 4.2}

It is straightforward but rather tedious to show that $e\left(\pi_{t}\right)$ is increasing in $\sigma_{1}^{2}$ and decreasing in $\sigma_{0}^{2}$. We therefore omit the details.

\section{Proof of Proposition 4.3}

As $\hat{\Sigma}_{1}\left(\pi_{t}\right)^{2}<\hat{\Sigma}_{0}\left(\pi_{t}\right)^{2}$, the entropy $e\left(\pi_{t}\right)$ is easily seen to be decreasing in the ratio $\hat{\Sigma}_{1}\left(\pi_{t}\right)^{2} / \hat{\Sigma}_{0}\left(\pi_{t}\right)^{2}$. This ratio increases with $N \gamma_{t}$, which in turn can be shown to increase with $N$.

\section{Proof of Proposition 4.4}

With probability one, none of the actions chosen by informed traders in the linear equilibrium is "confounding" (i.e. completely uninformative) in the sense of Easley and Kiefer (1988). By a straightforward extension of Easley and Kiefer (1988, Theorem 8, p. 1057) to a multi-agent setting, therefore, the posterior belief at the end of the last trading round, $\pi_{T+1}$, converges to the "truth" with probability one as $T \rightarrow \infty$.

\section{Proof of Proposition 5.2}

To ease notation, we suppress the dependence of various quantities on the current belief $\pi_{t}$. Conditional on $\sigma_{u}^{2}=\sigma_{k}^{2}$, the variables $v_{t}$ and $z_{t}=\lambda_{k, t}\left(N \beta_{t} v_{t}+u_{t}\right)$ are jointly normal with $\mathrm{E}\left[v_{t} \mid \sigma_{u}^{2}=\sigma_{k}^{2}\right]=\mathrm{E}\left[z_{t} \mid \sigma_{u}^{2}=\sigma_{k}^{2}\right]=0, \operatorname{Var}\left[v_{t} \mid \sigma_{u}^{2}=\sigma_{k}^{2}\right]=\sigma_{v}^{2}, \operatorname{Var}\left[z_{t} \mid \sigma_{u}^{2}=\right.$ $\left.\sigma_{k}^{2}\right]=\lambda_{k, t}^{2}\left(N^{2} \beta_{t}^{2} \sigma_{v}^{2}+\sigma_{k}^{2}\right)$ and $\operatorname{Cov}\left[v_{t}, z_{t} \mid \sigma_{u}^{2}=\sigma_{k}^{2}\right]=\lambda_{k, t} N \beta_{t} \sigma_{v}^{2}$. By the Projection Theorem,

$$
\operatorname{Var}\left[v_{t} \mid \sigma_{u}^{2}, z_{t}\right]=\operatorname{Var}\left[v_{t} \mid \sigma_{u}^{2}\right]-\frac{\operatorname{Cov}\left[v_{t}, z_{t} \mid \sigma_{u}^{2}\right]^{2}}{\operatorname{Var}\left[z_{t} \mid \sigma_{u}^{2}\right]},
$$

hence

$$
\operatorname{Var}\left[v_{t} \mid \sigma_{u}^{2}, z_{t}\right]=\sigma_{v}^{2}-\frac{N^{2} \beta_{t}^{2} \sigma_{v}^{4}}{N^{2} \beta_{t}^{2} \sigma_{v}^{2}+\sigma_{u}^{2}}=\sigma_{v}^{2} \frac{\sigma_{u}^{2}}{N \gamma_{t}+\sigma_{u}^{2}},
$$

with the last equality following from equation (7). 
Since $\mathrm{E}\left[v_{t} \mid \sigma_{u}^{2}, z_{t}\right]=z_{t}$ by (11), we have $\operatorname{Var}\left[\mathrm{E}\left[v_{t} \mid \sigma_{u}^{2}, z_{t}\right] \mid \pi_{t}, z_{t}\right]=0$, so the Law of Iterated Variances reduces to $\operatorname{Var}\left[v_{t} \mid \pi_{t}, z_{t}\right]=\mathrm{E}\left[\operatorname{Var}\left[v_{t} \mid \sigma_{u}^{2}, z_{t}\right] \mid \pi_{t}, z_{t}\right]$. In Section 4.3 we calculated the conditional probability $\hat{\pi}_{t}$ that $\sigma_{u}^{2}=\sigma_{1}^{2}$ given $z_{t}$ and the belief $\pi_{t}$ held at the beginning of trading round $t$. By (A.7), we then have

$$
\begin{aligned}
\operatorname{Var}\left[v_{t} \mid \pi_{t}, z_{t}\right] & =\sigma_{v}^{2} \mathrm{E}\left[\frac{\sigma_{u}^{2}}{N \gamma_{t}+\sigma_{u}^{2}} \mid \pi_{t}, z_{t}\right] \\
& =\sigma_{v}^{2}\left[\hat{\pi}_{t} \frac{\sigma_{1}^{2}}{N \gamma_{t}+\sigma_{1}^{2}}+\left(1-\hat{\pi}_{t}\right) \frac{\sigma_{0}^{2}}{N \gamma_{t}+\sigma_{0}^{2}}\right],
\end{aligned}
$$

which is strictly increasing in $\hat{\pi}_{t}$. Straightforward algebra using (9) shows that $\operatorname{Var}\left[v_{t} \mid \pi_{t}, z_{t}\right]=\sigma_{v}^{2} /(N+1)$ if and only if $\hat{\pi}_{t}=\pi_{t}$. By equation (20), $\hat{\pi}_{t}$ is strictly decreasing in $\left|z_{t}\right|$, so there is a unique cutoff level $\left|z_{t}\right|$ for the absolute price innovation such that $\hat{\pi}_{t}=\pi_{t}$ if and only if $\left|z_{t}\right|=\left|\bar{z}_{t}\right|$, and $\operatorname{Var}\left[v_{t} \mid \pi_{t}, z_{t}\right]>\sigma_{v}^{2} /(N+1)$ if and only if $\left|z_{t}\right|<\left|\bar{z}_{t}\right|$. Finally, note that $\mathrm{E}\left[\hat{\pi}_{t} \mid \pi_{t}\right]=\pi_{t}$, hence $\mathrm{E}\left[\operatorname{Var}\left[v_{t} \mid \pi_{t}, z_{t}\right] \mid \pi_{t}\right]=\sigma_{v}^{2} /(N+1)$ by equation (A.8) and the remark immediately after it.

\section{Proof of Proposition 5.3}

By Lemma 3.1 and the definition of $\bar{\sigma}_{t}^{2}$ in equation (5), we have $\Sigma_{t}^{I}=N \beta_{t} \sigma_{v}, \Sigma_{t}^{U}=\sqrt{\bar{\sigma}_{t}^{2}}$ and $\Sigma_{t}^{M}=\sqrt{N^{2} \beta_{t}^{2} \sigma_{v}^{2}+\bar{\sigma}_{t}^{2}}$. Since $\beta_{t}$ and $\bar{\sigma}_{t}^{2}$ are increasing in $\pi_{t}$, so is $\Sigma_{t}=\Sigma_{t}^{I}+\Sigma_{t}^{U}+\Sigma_{t}^{M}$. The result thus follows from Propositions 3.2 and 4.1.

\section{Proof of Proposition 5.4}

Define

$$
\eta_{k, t}=\left.\frac{\partial \gamma_{t}}{\partial \sigma_{k}^{2}}\right|_{\bar{\sigma}_{t}^{2}=\text { constant }}
$$

for $k=0,1$. Differentiating equation (9) with respect to $\sigma_{k}^{2}$ while holding $\bar{\sigma}_{t}^{2}$ fixed, we obtain

$$
\left(2 N \gamma_{t}+\sigma_{k}^{2}\right) \eta_{k, t}=\sigma_{k^{\prime}}^{2}-\gamma_{t}
$$

where $k^{\prime}$ is the element of $\{0,1\}$ different from $k$. At non-degenerate beliefs, $\sigma_{0}^{2}<\gamma_{t}<$ $\sigma_{1}^{2}$, hence $\eta_{1, t}<0<\eta_{0, t}$. The proposition now follows from Lemma 3.1.

\section{Proof of Proposition 5.6}

We will show that for $\sigma_{1}^{2} \leq 3 \sigma_{0}^{2} / N$, the function $\sqrt{\gamma}$ is convex. As the informed traders' beliefs follow a martingale, this will imply that $\beta_{t}=\sqrt{\gamma\left(\pi_{t}\right)} / \sigma_{v}$ is a submartingale by Jensen's inequality. The submartingale property for expected profits then follows from the fact that the these profits are proportional to trading agressiveness. 
The second derivative of $\sqrt{\gamma}$ is

$$
\frac{\gamma^{\prime}}{2 \sqrt{\gamma}}\left(\frac{\gamma^{\prime \prime}}{\gamma^{\prime}}-\frac{\gamma^{\prime}}{2 \gamma}\right),
$$

which has the same sign as the term in parentheses. Tedious but straightforward algebra shows that this term in turn has the same sign as $2 c(\pi)+\sqrt{c(\pi)^{2}+4 \sigma_{0}^{2} \sigma_{1}^{2}}$ where $c(\pi)=\sigma_{0}^{2}+\sigma_{1}^{2}-(N+1)\left[\pi \sigma_{1}^{2}+(1-\pi) \sigma_{0}^{2}\right]$ is the coefficient of $\gamma$ in the quadratic equation (9) for $\pi_{t}=\pi$.

Therefore, the second derivative of $\sqrt{\gamma}$ is positive at $\pi$ if and only if $c(\pi)>0$ (which is the case for $\pi$ close to 0 , but not for $\pi$ close to 1 ) or $0<-c(\pi)<2 \sqrt{N / 3} \sigma_{0} \sigma_{1}$. As $c(\pi)$ is strictly decreasing in $\pi$, the function $\sqrt{\gamma}$ is thus convex throughout if and only if $-c(1)<2 \sqrt{N / 3} \sigma_{0} \sigma_{1}$. With $-c(1)=N \sigma_{1}^{2}-\sigma_{0}^{2}$, one sees easily that this condition is equivalent to $\sigma_{1}^{2} \leq 3 \sigma_{0}^{2} / N$. 


\section{References}

Admati, A.R., 1991, "The Informational Role of Prices: A Review Essay," Journal of Monetary Economics, 28, 347-361.

Admati, A.R., and P. Pfleiderer, 1988, "A Theory of Intraday Patterns: Volume and Price Variability," Review of Financial Studies 1, 3-40.

Back, K., 1992, "Insider Trading in Continuous Time," Review of Financial Studies, $5,387-410$.

Back, K., H. Cao and G. Willard, 1998, "Imperfect Competition Among Informed Traders," Journal of Finance, forthcoming.

Bertsimas, D., and A. Lo, 1998, "Optimal Control of Execution Costs," Journal of Financial Markets, 1, 1-50.

Bolton, P., and C. Harris, 1999, "Strategic Experimentation," Econometrica, 67, 349374.

Carhart, M., 1997, "On the Persistence of Mutual Fund Performance," Journal of Finance, 52, 57-82.

Chan, L., and J. Lakonishok, 1993, "Institutional Trades and Intra-Day Stock Price Behavior," Journal of Financial Economics, 33, 173-199.

Chan, L., and J. Lakonishok, 1995, "The Behavior of Stock Prices Around Institutional Trades," Journal of Finance, 50, 1147-1174.

Easley, D., and N.M. Kiefer, 1988, "Controlling a Stochastic Process with Unknown Parameters," Econometrica, 56, 1045-1064.

Ellison, S.F., and W.P. Mullin, 1997, "Gradual Incorporation of Information into Stock Prices: Empirical Strategies," NBER Working Paper 6218.

Fama, E., 1991, "Efficient Capital Markets: II," Journal of Finance, 46, 1575-1617.

Forster, M.M., and T.J. George, 1992, "Anonymity in Securities Markets," Journal of Financial Intermediation, 2, 168-206.

Foster, F.D., and S. Viswanathan, 1990, "A Theory of Interday Variations in Volume, Variance, and Trading Costs in Securities Markets," Review of Financial Studies 3, 593-624.

Foster, F.D., and S. Vishwanathan, 1996, "Strategic Trading when Agents Forecast the Forecasts of Others," Journal of Finance, 51, 1437-1478. 
Gervais, S., 1997, "Market Microstructure with Uncertain Information Precisions: A New Framework," mimeo, University of Pennsylvania.

Gompers, P.A. and A. Metrick, 1998, "How are Large Institutions Different from Other Investors? Why do these Differences Matter for Equity Prices and Returns?" mimeo, Harvard Business School.

Grossman, S.J., 1976, "On the Efficiency of Competitive Stock Markets Where Traders Have Diverse Information," Journal of Finance, 31, 573-585.

Hausman, J., A. Lo and C. MacKinlay, 1992, "An Ordered Probit Analysis of Transaction Stock prices," Journal of Financial Economics, 31, 319-379.

Holden, C., and A. Subrahmanyam, 1992, "Long-lived Private Information and Imperfect Competition," Journal of Finance, 47, 247-270.

Holthausen, R., R. Leftwich and D. Mayers, 1987, "The Effect of Large Block Transactions on Security Prices: A Cross-Sectional Analysis," Journal of Financial Economics, 19, 237-267.

Holthausen, R., R. Leftwich and D. Mayers, 1990, "Large Block Transactions, the Speed of Response and Temporary and Permanent Stock-Price Effects," Journal of Financial Economics, 26, 71-95.

Jensen, M.C., 1969, "Risk, the Pricing of Capital Assets, and Evaluation of Investment Portfolios," Journal of Business, 42, 167-247.

Keim, D. and A. Madhavan, 1995, "Anatomy of the Trading Process: Empirical Evidence on the Behavior of Institutional Traders," Journal of Financial Economics, 37, 371-398.

Keim, D., and A. Madhavan, 1996, "The Upstairs Market for Large-Block Transactions: Analysis and Measurement of Price Effects," Review of Financial Studies, $9,1-36$.

Keller, G., and S. Rady, 1999, "Optimal Experimentation in a Changing Environment," Review of Economic Studies, 66, 475-507.

Kiefer, N.M., 1989, "A Value Function Arising in the Economics of Information," Journal of Economic Dynamics and Control, 13, 201-223.

Kraus, A., and H. Stoll, 1972, "Price Impacts of Block Trading on the New York Stock Exchange," Journal of Finance, 27, 569-588.

Kumar, P. and D. Seppi, 1994, "Information and Index Arbitrage," Journal of Business, 67, 481-509. 
Kyle, A., 1985, "Continuous Auctions and Insider Trading," Econometrica, 53, 13151336.

Leach, J.C., and A. Madhavan, 1993, "Price Experimentation and Security Market Structure," Review of Financial Studies, 6, 375-404.

Lindsey, R., 1992, "Market Makers, Asymmetric Information and Price Formation," unpublished Ph.D. thesis, Haas School of Management, University of California at Berkeley.

O'Hara, M., 1995, Market Microstructure Theory, Blackwell Publishers, Cambridge MA.

Perold, A., 1988, "The Implementation Shortfall: Paper versus Reality," Journal of Portfolio Management 14, 4-9.

Reiss, P.C., and I.M. Werner, 1998, "Does Risk Sharing Motivate Interdealer Trading?," Journal of Finance, 53, 1657-1704.

Scholes, M., 1972, "The Market for Securities: Substitution versus Price Pressure and the Effects of Information on Share Prices," Journal of Business, 45, 179-211.

Schwartz, R., and J. Shapiro, 1992, "The Challenge of Institutionalism for the Equity Markets," mimeo, New York University.

Seppi, D., 1990, "Equilibrium Block Trading and Asymmetric Information," Journal of Finance, 45, 73-94.

Spiegel, M. and A. Subrahmanyam, 1999, "Asymmetric Information and Disclosure Rules," mimeo, Yale School of Management.

Vayanos, D., 1998, "Strategic Trading in a Dynamic Noisy Market," mimeo, MIT. 